\title{
STRUCTURAL OPTIMISATION FOR DAMAGE TOLERANCE
}

\author{
$\underline{\text { Rui O. S. S. da Costa }}{ }^{{ }^{*}}$, Silvestre T. Pinho ${ }^{1}$ \\ ${ }^{1}$ Department of Aeronautics, Imperial College London, London SW7 2AZ, United Kingdom \\ *r.costa18@imperial.ac.uk
}

The manufacturing of composite structures can lead to small material imperfections (e.g. interplie debonding and small cracks) which current non-destructive measurement techniques are not able to detected. Therefore, it is of extreme importance to account for the presence of cracks or damage in the design stage such that the final optimised design is more robust and less sensitive to imperfections that may be missed during structural inspection.

Structural design and analysis are traditionally separated as being two distinct subjects. The need for faster time-to-market, more competitive pricing and greater structural performance creates the need for innovative approaches to design. To this end, approaches which combine both design and analysis can convert an otherwise iterative process into a seamless concurrent one. These approaches allow for the consideration of embedded cracks during the design; moreover, they create the opportunity to optimise the design for damage tolerance.

Structural optimisation is a family of methodologies that aim to provide a seamless design and analysis experience through the evolution and optimisation of design variables and parameters. Topology optimisation is one of such methodologies which focuses on obtaining the best distribution of material along the domain.

In this work, we propose a new methodology (see Figure 1) capable of modelling any evolving boundary and show how we can use this to optimise the topology of a structure considering the presence of cracks in the domain. We show that, making use of the explicitly defined boundary, we achieve improved accuracy and numerical efficiency, in comparison to implicit methods, through new velocity-extension and LSM-reinitialization procedures.

Furthermore, our methodology is successfully applied to the solution of several topology optimisation problems, some with embedded cracks in the initial domain (see Figure 2). We observe greater geometrical accuracy, leading to better stress-field predictions, as well as improvements in the optimum solutions when compared to implicit methods.

This method can potentially be applied to the design of damage-tolerant composite structures including skin-stiffener run-outs.

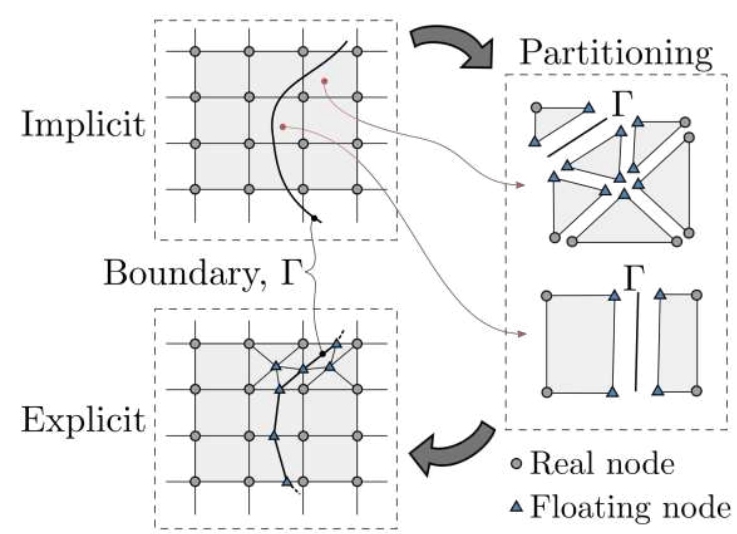

Figure 1. Partition of a quadrilateral mesh such that it conforms to a moving boundary

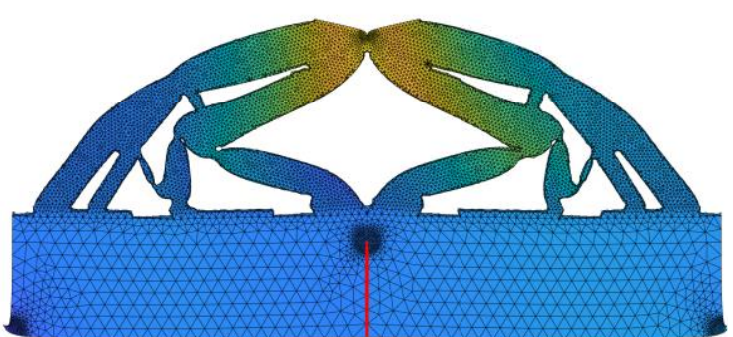

Figure. 2. Displacement of an optimised topology of an MBB beam with an embedded crack (red) 